\title{
Anabases
}

Mise au point en réponse à Jean-Marie Pailler, « Enseigner l'histoire des religions. Que faire de l'Antiquité ? À propos d'expériences et de publications récentes »(Anabases 2)

\section{Philippe Borgeaud}

\section{OpenEdition}

\section{Journals}

Édition électronique

URL : http://journals.openedition.org/anabases/3010

DOI : $10.4000 /$ anabases.3010

ISSN : 2256-9421

Éditeur

E.R.A.S.M.E.

Édition imprimée

Date de publication : 1 octobre 2006

Pagination : 261-262

ISSN : $1774-4296$

Référence électronique

Philippe Borgeaud, « Mise au point en réponse à Jean-Marie Pailler, « Enseigner l'histoire des religions. Que faire de l'Antiquité ? À propos d'expériences et de publications récentes » (Anabases 2) », Anabases [En ligne], 4 | 2006, mis en ligne le 01 janvier 2012, consulté le 21 octobre 2019. URL : http:// journals.openedition.org/anabases/3010 ; DOI : 10.4000/anabases.3010 


\section{Mise au point en réponse à Jean-Marie Pailler, \\ "Enseigner l'histoire des religions. Que faire de \\ l'Antiquité ? À propos d'expériences et de publications récentes " (Anabases 2)}

PHILIPPE BORGEAUD

Il APPARAîT À la LECTURE d'Anabases. Traditions et Réception de l'Antiquité, $\mathrm{n}^{\circ}$ 2, Toulouse 2005, que mon livre intitulé Aux origines de l'histoire des religions (Paris, Seuil, 2004) a eu l'heur d'irriter Jean-Marie Pailler. Cela resterait sans conséquence si le savant latiniste ne diffusait à mon sujet certaines insinuations d'ordre pédagogique, dans l'intention évidente d'écarter ma contribution du débat actuel qui porte sur l'enseignement de l'histoire des religions au niveau préuniversitaire, et sur la place que devraient occuper les religions antiques dans un tel enseignement. Je regrette cette tentative de mise à l'index, qui vient d'un savant dont je respecte bien évidemment les importants travaux (notamment sur les Bacchanales). Je laisse aux lecteurs plus avertis que moi du contexte général dans lequel évolue ce collègue, que je n’ai jamais rencontré, le soin d'imaginer quelle a bien pu être, au fond, la raison de son émotion, et je prie ceux qui auront réellement lu mon livre de bien vouloir comparer ce qui s'y trouve avec ce que Pailler en dit, dans son article intitulé "Enseigner l'histoire des religions. Que faire de l'Antiquité ? À propos d'expériences et de publications récentes ", p. 195-208 (précisément aux pages 199-204).

Pailler ne dit rien du cœur de mon livre, qui est une réflexion sur l'émergence du comparatisme entre hellénisme, judaïsme et christianisme, autour de la figure de Moïse. Il se contente d'affirmer que je suis une sorte de militant anti-chrétien radical, pour ne pas dire fondamentaliste, et que ce type d'attitude nuit à une approche calme et raisonnable de l'histoire des religions. Il tire cet argument du seul fait que j'en appelle à une attitude critique, historienne et anthropologique, comparatiste, distanciée. Mais alors pourquoi n'adresse-t-il pas le même reproche au théologien François Boespflug, éminent historien des religions dont il fait (à juste titre) l'éloge à propos de la même attitude, comparatiste et distanciée ? Poser la question revient à y répondre. On remarquera, de ce point de vue, qu'il a lu attentivement et avec plaisir René Girard, auquel il renvoie avec réserve certes, mais non sans ambiguïté (p. 201, avec note 15).

Aux pages 206-207 de mon livre, j’ai évoqué la nécessité de sortir du cadre pieux et mythologique, pour pouvoir pratiquer l'histoire des religions. J'ai défini (p. 183-213) ce que j'entendais par ce cadre. Pailler trouve cette mise en garde désuète, pour ne pas dire ridicule. Il ne croit pas un instant que l'ethnocentrisme (en l'occurrence le christiano-centrisme, le sien) puisse 
encore être un obstacle, de nos jours (voyons !), à la bonne observation de phénomènes religieux extérieurs à notre culture. Il a la chance de côtoyer, dans l'école laïque et républicaine, des esprits libres. Mais est-ce vraiment si simple?

Contrairement à ce que prétend Pailler, je n'ai jamais écrit qu'il fallait abandonner le mot religion. Encore moins lui substituer quoi que ce soit. Sa note 13, p. 200, est particulièrement absurde : il m'y reproche de vouloir substituer au mot religion la définition que j'en donne, une définition que j'élabore en m'inspirant des auteurs latins. Dire le sens que l'on accorde à un mot reviendrait ainsi à le condamner ? ! Le remplacement que je souhaite n'est pas celui du mot, auquel un autre (lequel ?) serait substitué, mais bien celui du sens qu'on lui donne aujourd'hui. Je plaide pour un retour au sens le plus ancien. Quant à ce sens, sur lequel Benveniste en effet projette une lumière de type étymologique (que l'étymologie soit vraie ou fausse peu me chaut), je me contente pour ma part de m'en tenir à l'usage, un usage pré-chrétien déjà relativement bien décrit dans l'article "religio " du Daremberg et Saglio. C'est cela qui me conduit (p. 206) à faire de l'histoire des religions l'" histoire des relectures et des choix (élections), histoire des scrupules, des hésitations, histoire des rites et des discours tâtonnants qui les accompagnent ».

Contrairement toujours à ce que Pailler écrit (p. 201), je n'ai pas opposé l'intolérance chrétienne à la tolérance païenne. Je n'ignore ni le procès de Socrate, ni l'affaire des Bacchanales. Mais je n'en fais pas des affaires réductibles au seul champ religieux. J'ai simplement rappelé, après beaucoup d'autres, que la prétention de détenir une vérité révélée et qui plus est de nature difficilement compatible avec les croyances et les pratiques des autres pose un problème supplémentaire. Cette prétention, en effet, n'est pas particulièrement propice au développement de la tolérance. Mais j'ai situé l'émergence de cette attitude chrétienne sur l'horizon d'autres violences, et plus précisément en référence aux discours accusateurs qui font des autres (notamment des Judéens, dans une certaine idéologie égyptienne, puis hellénistique), des athées ou des iconoclastes. J'ai parlé à ce propos de violences égyptiennes, grecques et perses, romaines aussi. Comme je l'ai écrit à la page 211, j'ai voulu « rendre compte d'une manière théorique de ce qui fut une vive controverse, parfois violente, impliquant des Égyptiens, des Grecs et des Judéens », rendre compte d'une « altercation ». Le christianisme émergeant (dont j'affirme en effet que certaines manifestations furent plus radicales, en termes de rupture, que le judaïsme), je me suis permis de porter sur lui un regard critique dans la seule mesure où, je le rappelle, l'histoire comparée des religions est une invention chrétienne. Dans cette mesure la critique est nécessaire, préalable à toute expérience d'ordre scientifique. C'est dans cette mesure, et elle seule, que je suis concerné en tant qu'historien des religions, plus probablement que ne l'est Jean-Marie Pailler qui ne me semble pas être, lui, particulièrement comparatiste.

Enfin, foi de mécréant, mes ressources méthodologiques, contrairement encore à ce que soupçonne Jean-Marie Pailler (note 13, p. 200 ; et p. 204), ne sont ni Descartes, ni Auguste Comte.

Philippe Borgeaud

Université de Genève

Faculté des Lettres

3, rue de Candolle

CH - 1211 Genève 4

philippe.borgeaud@lettres.unige.ch 